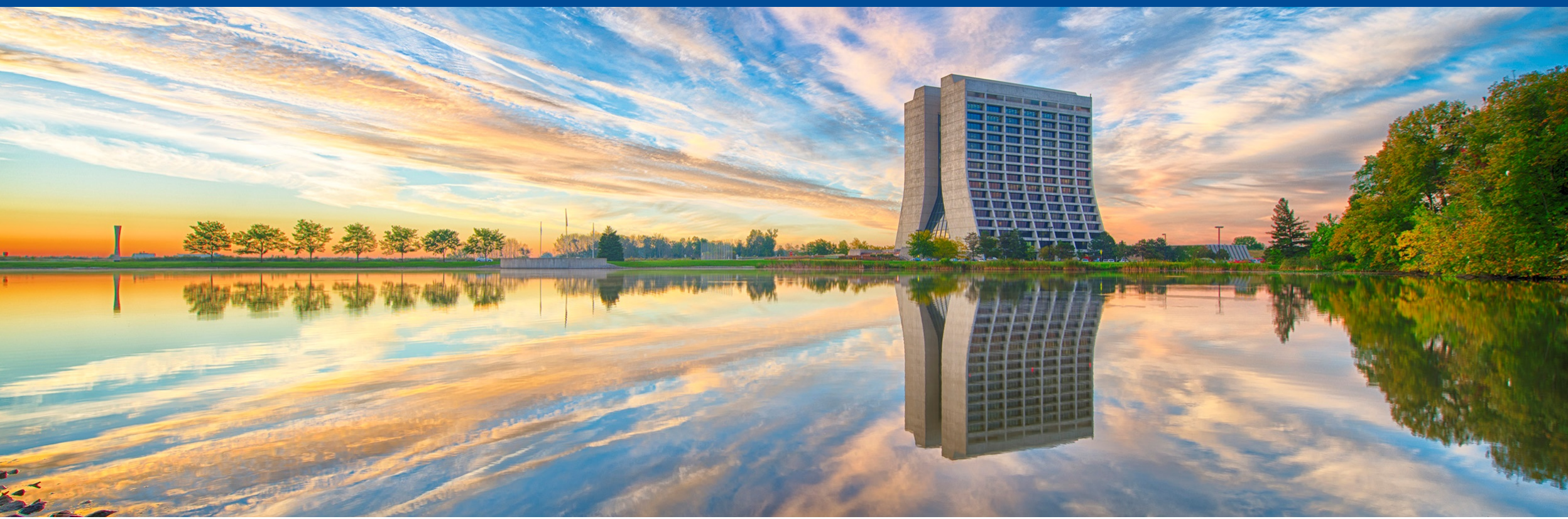

\title{
Design Considerations and Operational Features of the Collimators for the Fermilab Main Injector and Recycler
}

[12 years in 12 minutes]

Bruce C. Brown

Main Injector Department, Fermi National Accelerator Laboratory 4 September 2019 WEYBB6 at NAPAC2019

This manuscript has been authored by Fermi Research Alliance, LLC under Contract No. DE-AC02-07CH11359 with the U.S. Department of Energy, Office of Science, Office of High Energy Physics. 


\section{Outline}

Purpose

Design

Implementation

Operation

Results

Lessons Learned 


\section{Fermi National Accelerator Lab -- Accelerators}

Collimation Systems:

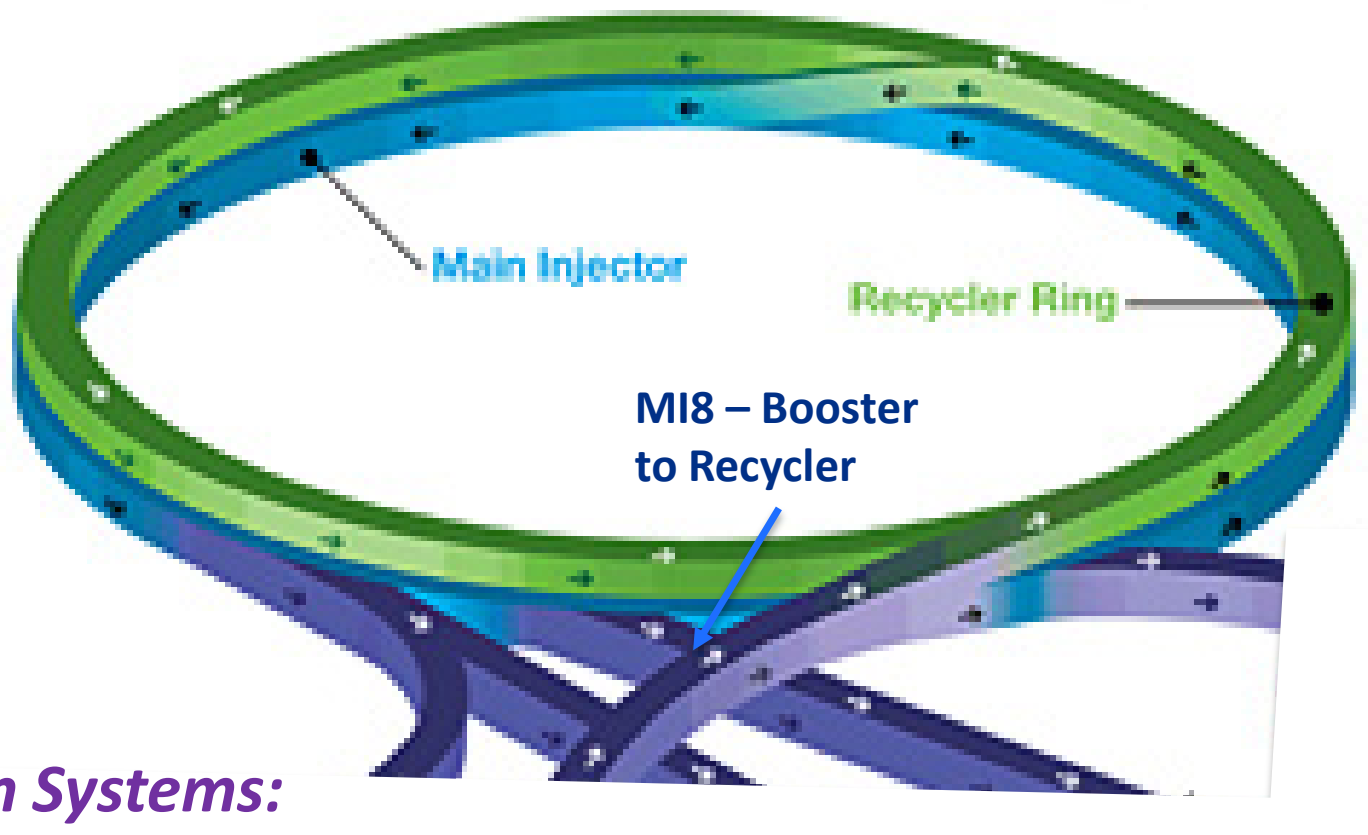

MI8 Transfer Line -

Four 4-Ton Collimators - Installed Spring 2006

Main Injector -

Primary + Four 20-Ton Secondary + 6 Masks - Installed Fall 2007

Additional Masks added in subsequent shutdowns

Recycler-

Primary + Two 20-Ton Secondary + 2 Masks - Installed Summer 2016 


\section{Why Collimate?}

Purpose for Collimation

Localize beam loss in a dedicated region, reducing activation and component degradation in the rest of the ring

Radiation damage proportional to kinetic energy of lost protons.

$700 \mathrm{~kW}$ of $120 \mathrm{GeV}$ protons delivered. $50 \mathrm{~kW}$ of $8 \mathrm{GeV}$ protons

With 1\% loss (500 W) in transfer line, 3\% loss (1500 W) in Recycler,

3\% loss (1500 W) in MI.

All loss at or near $8 \mathrm{GeV}$ injection energy. Losses must be localized.

Beam Losses occur at aperture restrictions due to

Transverse Tails

Beam out of RF Acceleration Bucket

Instabilities

Beam properties change, apertures remain until changed. 


\section{Loss Monitoring}

Why do losses change?

1. Booster emittance changes (transverse or longitudinal)

2. RF System performance (insufficient bucket area)

3. Instabilities

How do we know?

1. Beam Loss Monitors are at each quad plus at transfer points

2. Residual Radiation is in proportion to nearby BLM signal

3. Loss Monitors are displayed in control room

4. Residual Radiation is recorded during accesses

Loss History defines Radiation History (HB2010 Paper)

1. At any loss point, activation is proportional to nearby BLM

2. Residual Radiation predicted by half life weighted BLM history

3. MARS studies identify isotopes to include in weighted history 


\section{Design}

\section{Transfer Line Main Injector Recycler Collimators Collimators \\ Collimators}

One-Stage

Transverse (H\&V)

Four 4-Ton

Collimators

- Each Pair Scrapes in Two Opposite

Corners

Pairs are one cell $\left(90^{\circ}\right)$ apart

Taper Upstream
- Two Stage

- Uncaptured Beam

- Off-Momentum at $\sim 1 \% \delta p / p+H \& V$

Radial Edge Primary

- Four 20-Ton

Secondaries

- Near and Far Masks (fixed outside pipe)

- Taper Upstream
- Two Stage

- Transverse Tails

- Vertical Edge

- Two 20-Ton Secondaries

- Near Masks

- Some Coupling for Horizontal Tails

- Taper Upstream \& Downstream 


\section{MI8 Transfer Line 4-Ton Collimator Pair}

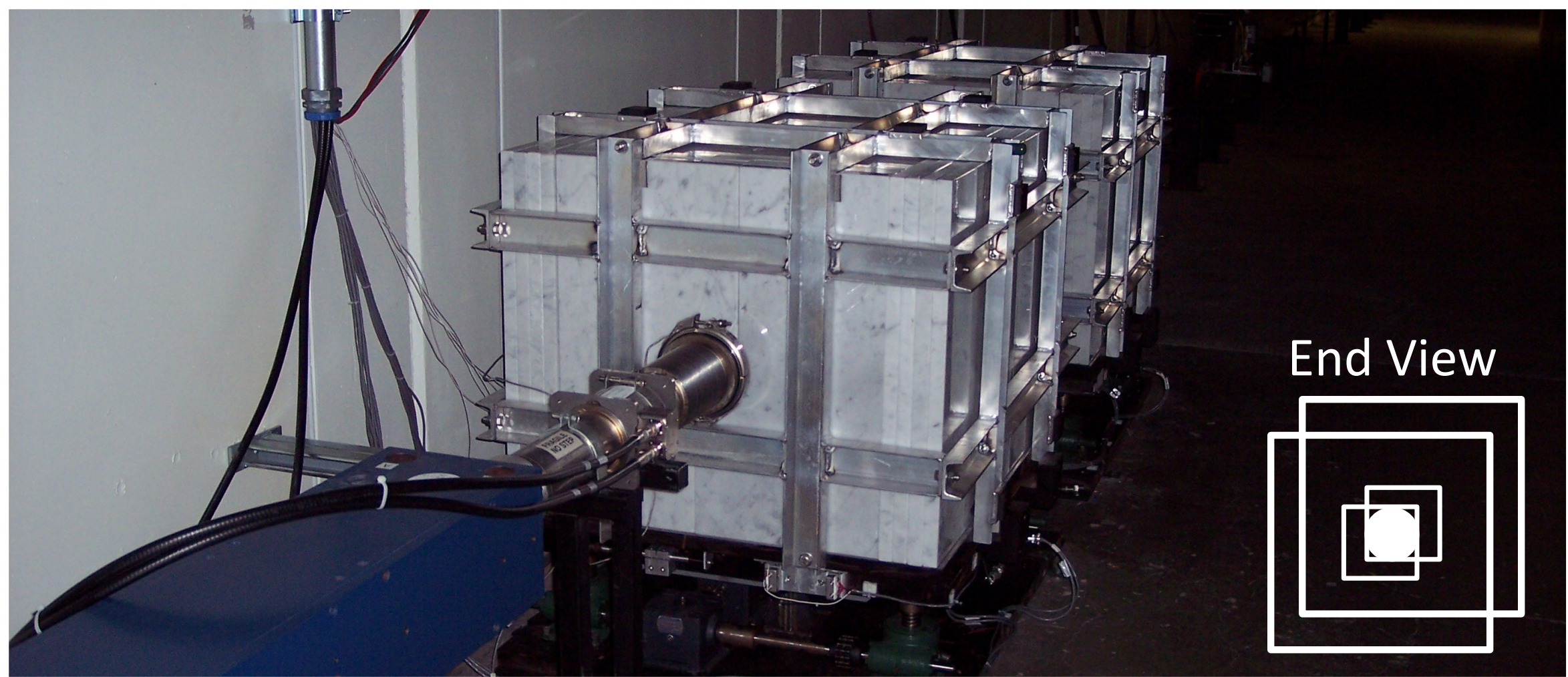

One collimator is positioned so that beam is in a corner to scrape $\mathrm{H}$ edge and $\mathrm{V}$ edge. Next Collimator is positioned to scrape the opposite corner.

Next Collimator Pair is $90^{\circ}$ phase advance away - angle edge becomes position edge. 


\section{Main Injector 20-Ton Secondary Collimator}

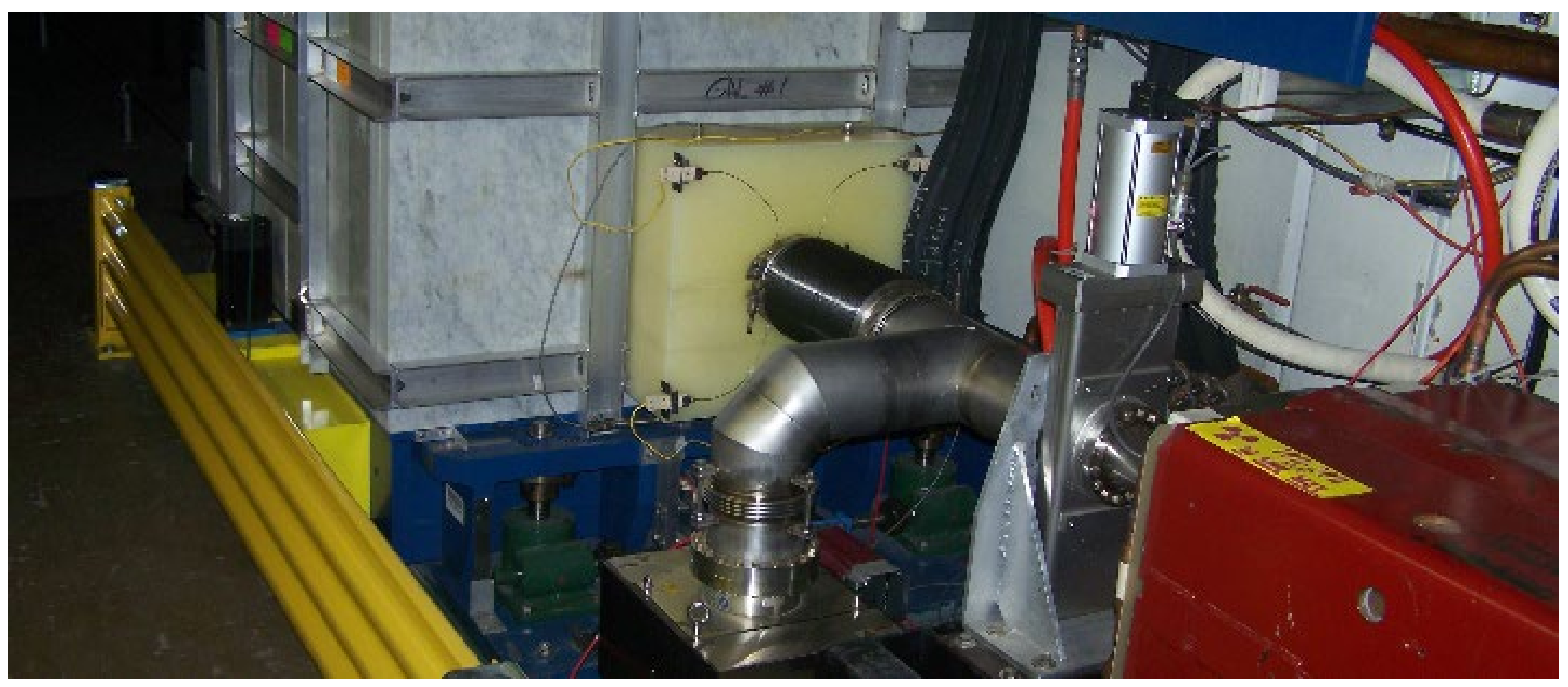

C301 20-Ton Secondary Collimator from Upstream.

Motion in $\mathrm{H}$ and $\mathrm{V}$ directions (no $\mathrm{H}$ angle, have not used $\mathrm{V}$ angle)

Transverse positions controlled by stepping motors with high precision

$\sim 12 \mathrm{~cm}$ of Marble surrounds steel to absorb residual radiation during access

$>23$ " (>58 cm) transverse space required to absorb shower with steel absorber 


\section{Recycler Collimators - Secondary and Mask}

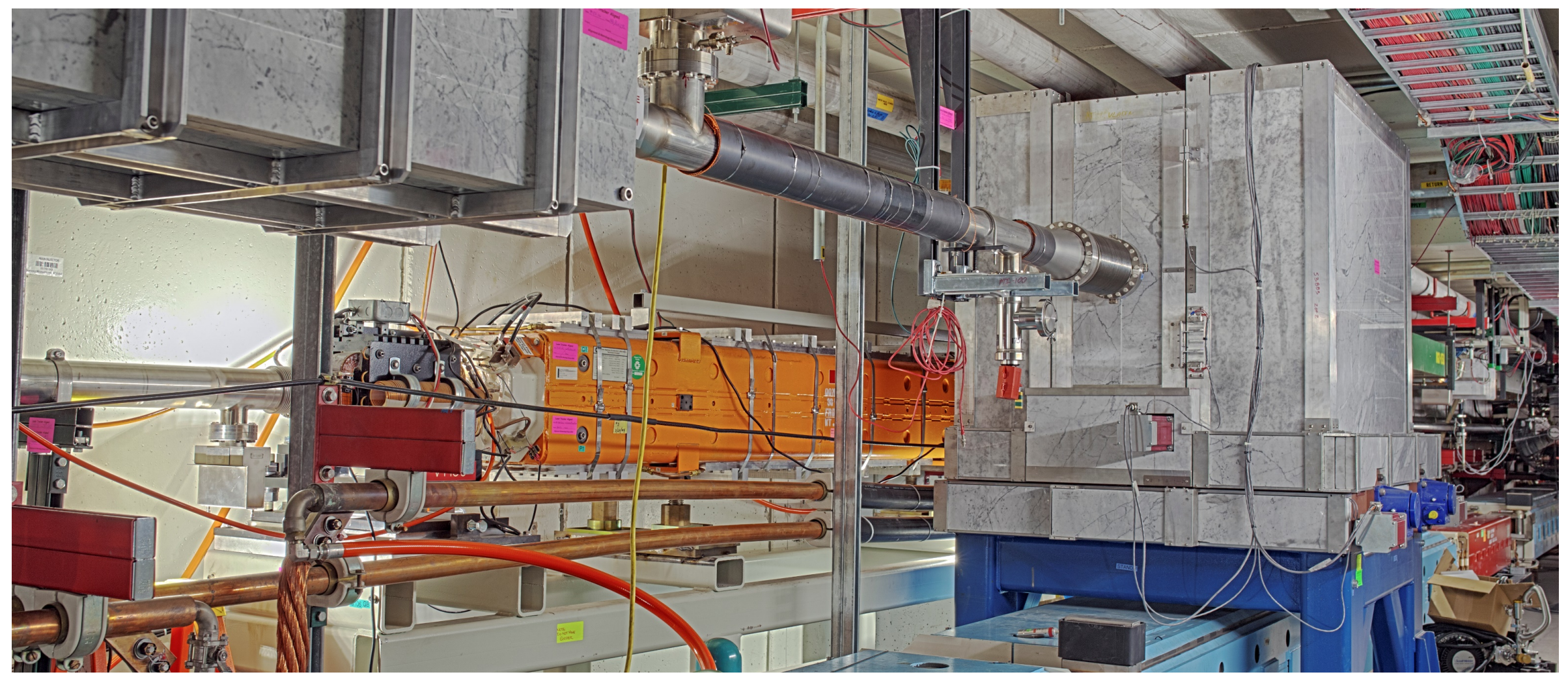

C613 20-Ton Collimator with mask. Primary Collimator visible upstream. Horizontal and Vertical Motion with $\mathrm{V}$ angle (pitch) and $\mathrm{H}$ angle (yaw using roller plates). Stepping motors for precision motion.

$>23$ " (>58 cm) transverse space required to absorb shower with steel absorber 


\section{Main Injector Collimation - Uncaptured Beam}

Slip Stacking provides double intensity but with some beam which is not in RF buckets.

As magnets ramps and beam Is accelerated, uncaptured beam is left to drift inside

A scattering foil is positioned to interact after $\sim 1 \%$ acceleration.

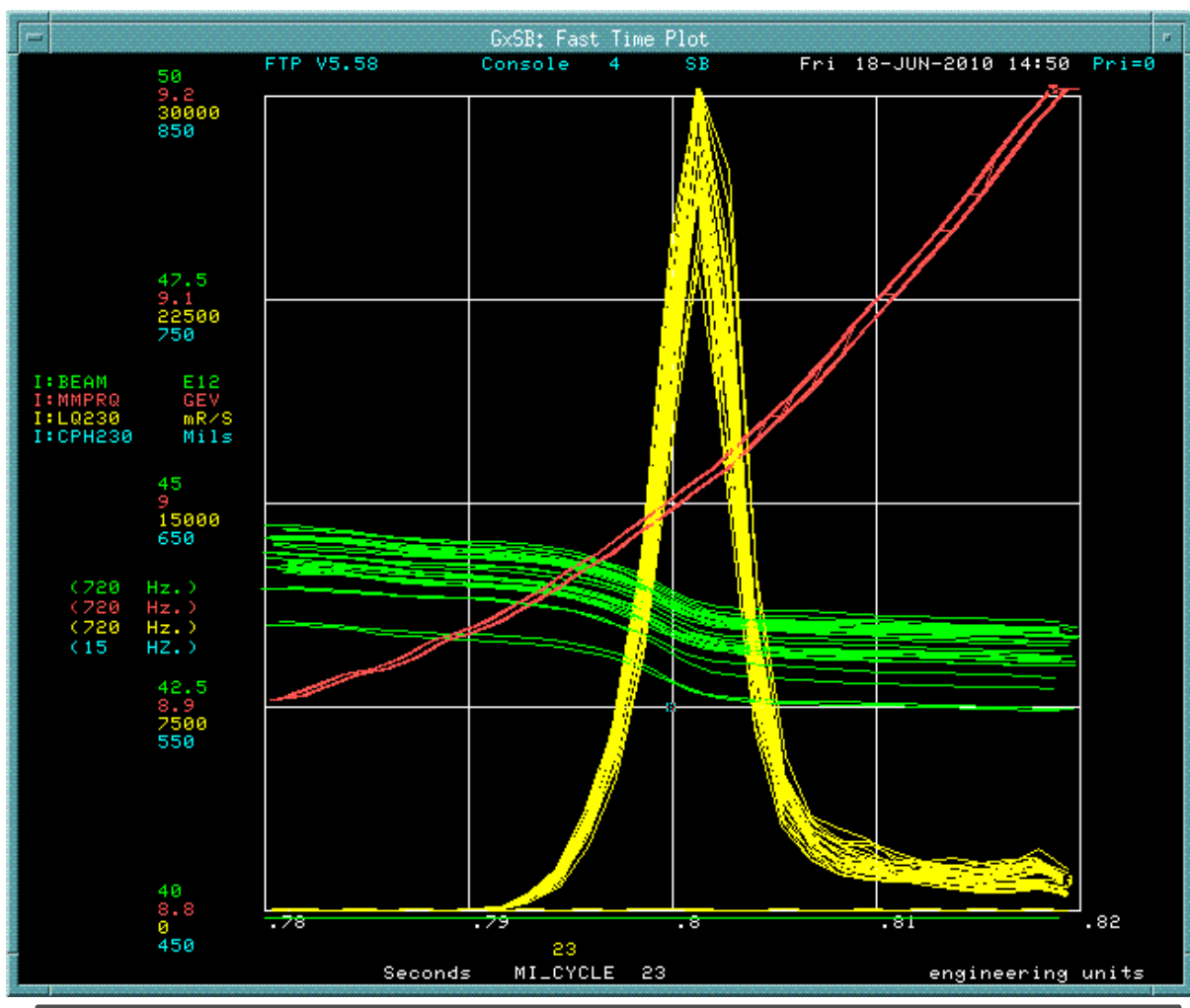
An orbit bump moves beam into it. Here we see Beam intensity, Momentum, and Loss ât Prơmary

$$
2-3 \% \text { loss } 1 \% \text { acceleration } 00 \text { in mSeconds }
$$




\section{Design-Primary-Secondary (two-stage) collimation}
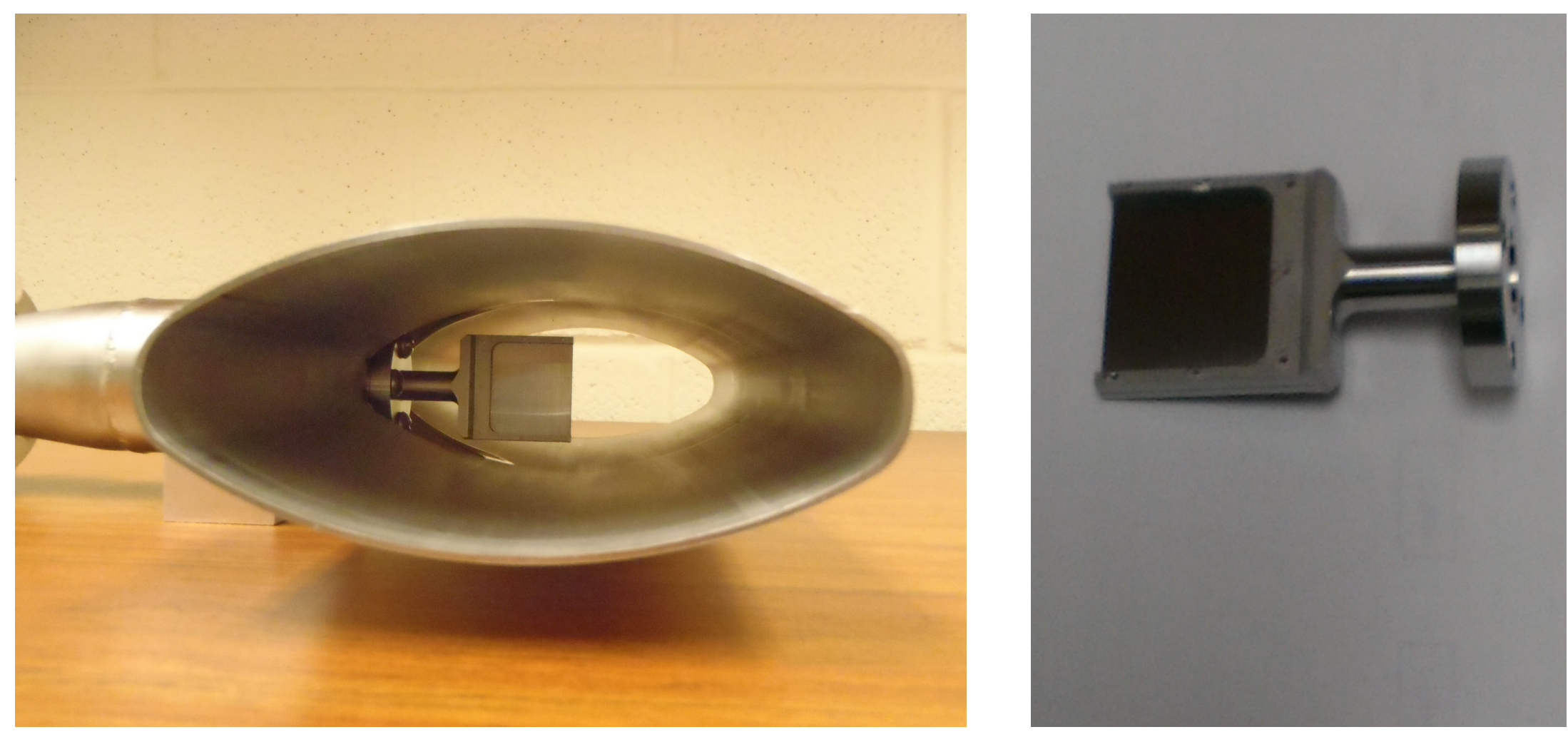

Primary Collimator $(0.010$ " $W)$ at 3 sigma on inside to scatter from $x$ edge. 


\section{Design-Primary-Secondary (two-stage) collimation}

Main Injector

Two-Stage

Collimation

On Average

Lost Protons

Strike Primary

2.5 Times

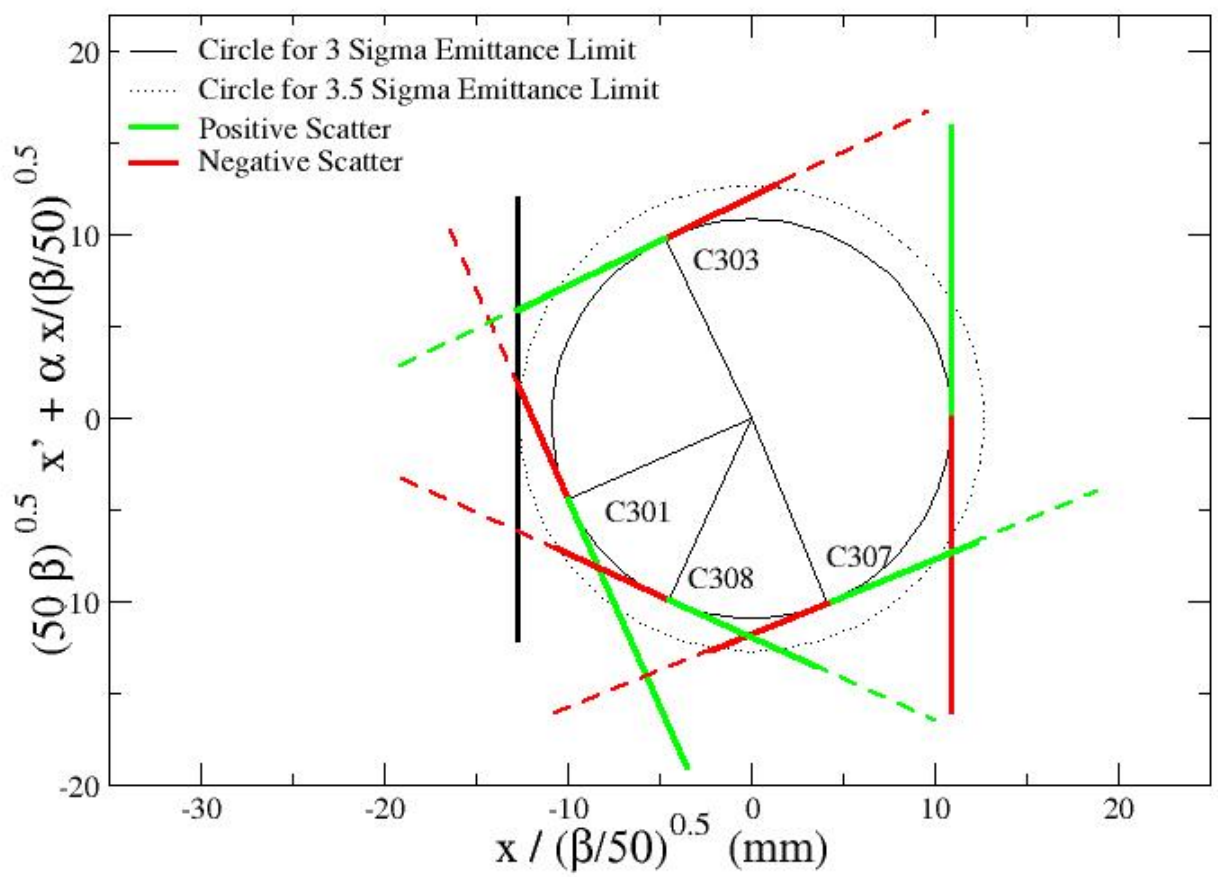

Two Stage Collimation in Reduced Coordinates (which preserve emittance of beam). Primary Collimator $(0.010$ " W) at 3 sigma on inside to scatter from $x$ edge. Secondary collimators at C301, C303, C307, C308 to scrape at either plus or minus x edge Tune collimation to place secondary collimators at about 3.5 sigma. 


\section{Operational Tuning}

MI8 -

Move Collimators to Beam Edge

Auto-tune to keep orbit.

$\mathrm{MI}-$

Place beam edge parallel to collimator

(Complicated Orbit Bump)

Move collimators to achieve containment.

RR -

Pitch and yaw provided (Simple orbit bump)

Move collimator to scrape edges in both planes

(but vertical scattering from primary)

Beam Loss Monitors (glass tubes with argon gas)

record losses to allow tuning and monitoring 


\section{Results: Excellent Loss Containment}

MI8 - Good Orbit stability for MI/RR Beam

Scrape $1 \%$ (at design limit)

Needed to correct $8 \mathrm{GeV}$ to Booster Neutrino Beam (BNB)

to avoid excess loss

MI - Use loss monitors to record loss - Study Uncaptured Loss $>99 \%$ Containment of Uncaptured (off momentum) beam loss recorded in Collimation Region in Main Injector (16 half cells out of 208 half cells) $>93 \%$ Containment of all loss MARS $\sim 80 \%$ of lost power absorbed in 20 -Ton secondaries

RR - Good containment. Damping of instabilities reduced loss. 


\section{Other Results -- Stainless Steel Pipe Damage}

Inserted collimators into an existing tunnel with cable plant. This included cables for the Electron cooling system.

During the 2012-13 upgrade and Subsequently we found chlorine-induced pitting corrosion. We ascribe that to the release of $\mathrm{Cl}$ from PVC cable jackets which are about $50 \% \mathrm{Cl}$.

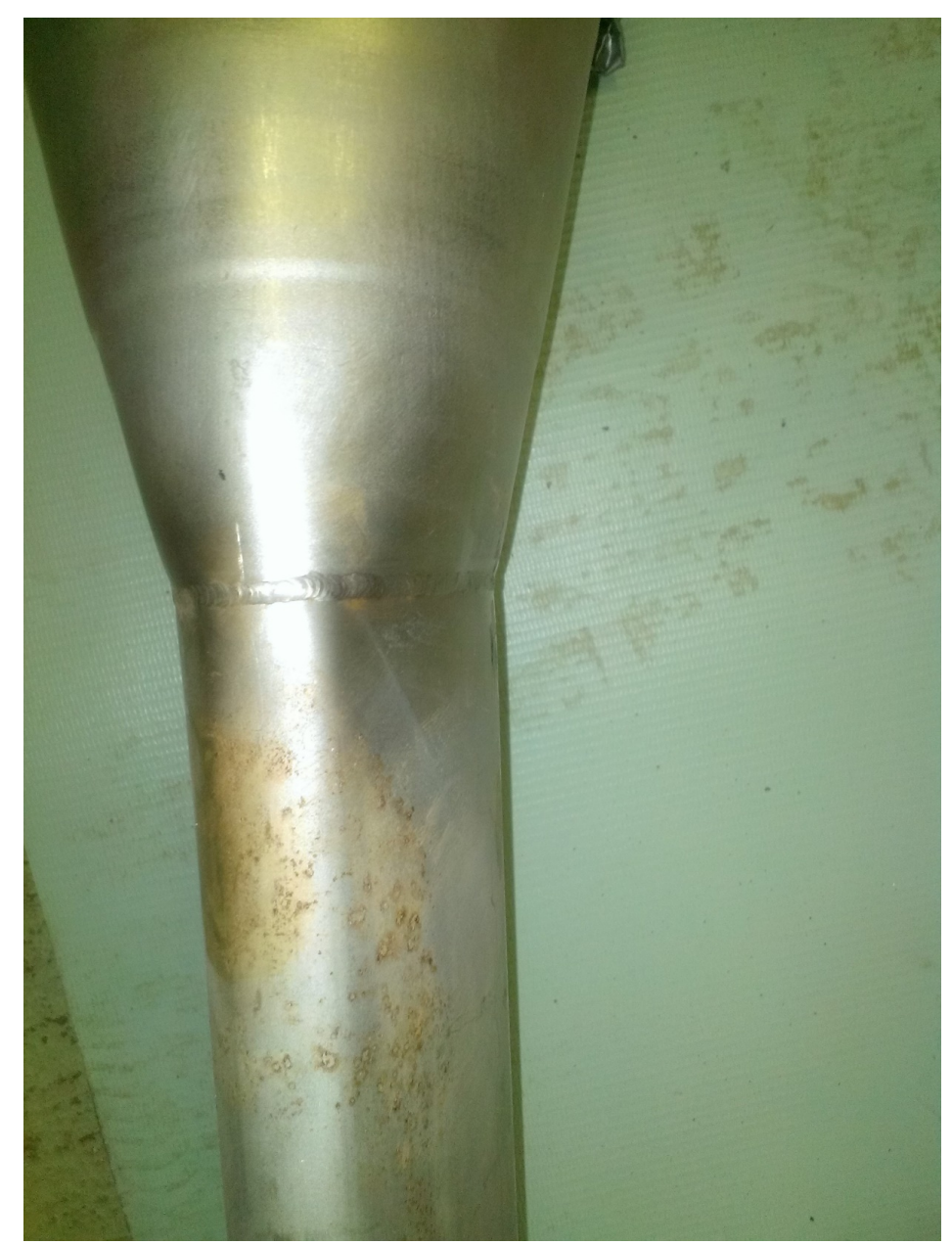




\section{Design Features to Reconsider}

Secondary Collimators Contain Shower Maximum Completely.

Tunnel depth is sufficient to contain Muon prompt dose Muons from $8 \mathrm{GeV}$ showers do not create activation.

One might consider motion control options which require space in the region with higher fluxes of hadrons.

We kept the mechanical system simple and rugged.

Vertical Angle (pitch) is easy enough if needed.

Horizontal Angle (yaw) required roller plates. Not as precise.

One Must Plan to Remove Secondaries without exposing high radioactivity portions 


\section{Further Progress -- Still learning - Cable Failure}

These collimators were inserted into an operating accelerator so only minimal allowance was made for accommodating them.

The cable plant includes signal cables, RG58 Red high voltage cables for beam loss monitors ( $2 \mathrm{kV}$ ) and ion pumps (5 kV).

Cables are in trays above the aisle but near the Main Ring.

We had no failures until 2016. By 2019, all $5 \mathrm{kV}$ cables have failed somewhere in the collimator region.

MARS studies combined with BLM integral loss suggest that failure occurred after $5500 \mathrm{~Gy}(0.55 \mathrm{Mrad})$ of absorbed dose. 


\title{
Co - Authors
}

Philip Adamson, Robert Ainsworth, David Capista, Kyle Hazelwood, loanis Kourbanis, Nikolai V. Mokhov, Denton K. Morris, Vitaly S. Pronskikh, Igor Rakhno, Igor S. Tropin, Meiqin Xiao, Ming-Jen Yang

\author{
Accelerator Division, \\ Fermilab, \\ Batavia, Illinois
}




\section{Acknowledgements}

- Special Thanks to Alexandr Drozhdin, Vladimir Sidorov, Kiyomi Seiya, Guan Wu for fundamental contributions to many aspects.

- Thanks for many people from the Accelerator Division, the Particle Physics Division and the rest of the lab for assistance in implementing these systems.

- Thanks to the Accelerator Division Operations Department for employing these systems while moving from $200 \mathrm{~kW}$ to $700 \mathrm{~kW}$ proton beams delivered from the Main Injector.

- We are grateful for the Experimenters who have made use of this beam and the Fermilab Management for sustaining the Fermilab High Energy Physics program.

- This research used, in part, an ALCC allocation at the Argonne Leadership Computing Facility, which is a DOE Office of Science User Facility supported under Contract DE-AC02-06CH11357. 


\section{Backup Slides}




\section{Lessons Learned}

Following 15 years of high intensity operations, we have several lessons learned from operations at up to $700 \mathrm{~kW}$ Proton power.

1. Two machines in one tunnel is at least as difficult as one imagines:

- One begins by solving physical interferences (and losing symmetry to do so).

- On must then make provision for monitoring such things as losses to separate issues in the two machines. Solutions which are suitable for studies may still leave issues for operational monitoring.

2. Aperture does matter: the Recycler has even smaller vertical aperture than the Main Injector. Lots of effort and TIME is required to learn to accommodate the Booster emittance. 


\section{Lessons Learned}

Following 15 years of high intensity operations, we have several lessons learned from operations at up to $700 \mathrm{~kW}$ Proton power.

3. With adequate (even if small) multiturn aperture, two-stage collimation is very effective.

4. As you need to account for smaller loss fractions, second order effects may not be small enough. For example, a small transverse loss for part of a turn may still accumulate.

5. Loss-induced activation may release $\mathrm{Cl}$ and $\mathrm{Cl}$ may attack stainless steel (chlorine-induced pitting corrosion). We have had vacuum pipe which would not hold rough vacuum.

6. Radiation-induced cable failure. Ion pumps powered at $5 \mathrm{kV}$ have experienced cable failure. Few failures in 12 years but nearly all failed after 15 years. $2 \mathrm{kV}$ loss monitor cables fail more slowly. 


\section{Lessons Learned}

Following 15 years of high intensity operations, we have several lessons learned from operations at up to $700 \mathrm{~kW}$ Proton power.

7. Heads-up displays for operations are valuable.

8. Instrumentation for routine operations will require trade-off's for dynamic range vs. resolution, time resolution vs. time range and current monitoring vs. data logging. When making choices for operations, seek to preserve flexibility (at the expense of convenience) for study requirements.

9. Things will fail. Try to consider failure modes (mechanical hard stops on motion systems), recovery options, and eventual removal at end of life.

10. Convenient cross checks (rulers or simple measurements) on motion systems have been helpful.Need $1 \mathrm{~mm}$ not 0.001 " 


\section{Lessons Learned}

Following 15 years of high intensity operations, we have several lessons learned from operations at up to $700 \mathrm{~kW}$ Proton power.

11. To minimize exposure for maintenance, a taper from the front of the collimator to the design interaction point is essential. Beam scattered at the primary collimator will typically be intercepted there. Since there will be losses which are not due to scattering in the primary, it is prudent to also provide a taper at the downstream end so that other loss-induced activation will be inside the secondary collimator.

12. For $8 \mathrm{Gev}$ proton loss, the outscattered protons and produced secondaries are very forward. The aperture for the secondary collimator should be minimized. 


\section{Abstract}

- The Fermilab Main Injector system delivers $700 \mathrm{~kW}$ of 120 GeV Proton beam for neutrino experiments. Since 2013 this has been achieved using slip stacking accumulation in the Recycler with up to 12 batches from the Fermilab Booster per Main Injector Ramp Cycle. To control activation from beam loss, collimation systems in the Booster to Recycler transfer line, in the Recycler and in the Main Injector are employed. Residual radiation measurements around the ring with detailed studies at the collimators are required to maintain adequate loss control. We will review design considerations, operational parameters and activation results for more than ten years of operation. Simulations with MARS15 are used to explore the activation rates and the isotopic composition of the resulting activation. 